\title{
A dynamic reward-based incentive mechanism: reducing the cost of P2P systems
}

\author{
Kun $\mathrm{Lu}^{\mathrm{a}}$, Shiyu Wang ${ }^{\mathrm{a}}$, Ling Xie ${ }^{\mathrm{a}}$, Zhen Wang ${ }^{\mathrm{a}, \mathrm{b}}$, Mingchu Li ${ }^{\mathrm{a}, *}$ \\ ${ }^{a}$ 116620, Software School, Dalian University of Technology, Liaoning Province, China \\ ${ }^{b}$ School of Computer Engineering, Nanyang Technological University, Singapore 639798
}

\begin{abstract}
Reward-based incentive mechanisms are used most commonly to promote cooperation in peer-to-peer (P2P) systems. Specifically, contributing agents are rewarded by the system. For a centralized P2P system, the central server is responsible for implementing the mechanism and rewarding those cooperative agents. Thus, providing incentives could be costly for the system server. In this paper, we propose a dynamic reward mechanism in which the reward changes accordingly as the system evolves, i.e., when there are more cooperative agents, the reward for each agent decreases, and when there are no free-riders, rewards are no longer needed. Through theoretical analysis using replication dynamics, we determined the evolution stable strategy (ESS) for different scenarios and qualitatively proved that our dynamic reward mechanism can reduce the overall cost of the system. The simulation proved that this dynamic reward mechanism can promote cooperation and reduce the cost of the system.
\end{abstract}

Keywords: incentive mechanism, dynamic reward, replicator dynamics, evolutionary game

\section{Introduction}

Peer-to-peer (P2P) communication technologies have been used exten3 sively in file sharing [I] and video streaming applications [2, 3] due to their 4 low bandwidth requirement and high flexibility. By definition, the stability

\footnotetext{
${ }^{*}$ Corresponding author.

Email address: mingchul@dlut.edu.cn (Mingchu Li)
} 
of such systems relies on the voluntary contributions of agents. However, the fundamental principle for these systems is to accommodate participants rational behaviors and design for choice [4]. These rational and strategic agents will choose to share only if they have better long-term benefits. This leads to the well-known free-riding problem [5], which degrades the performance of a P2P system tremendously.

Incentive mechanisms are used to solve the lack of cooperation problem in P2P systems [6]. Among these mechanisms, reward-based incentives are

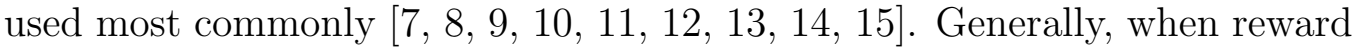
mechanisms are used, contributing agents may get additional rewards. Typically, reward mechanisms can be divided into two types, i.e., peer rewards and institution rewards [16]. For peer rewards, agents exist who voluntarily help to reward cooperative peers. But this method requires that agents have great power to recognize and reward others, which means it is not realistic in a real system. However, in the case of institution rewards, cooperative agents are rewarded by a central server. The server tracks peers behaviors and rewards them accordingly. However, since the system gives the rewards in the institution reward mechanism, doing so can be costly to the server. Thus, saving costs in the system under the premise of enhancing cooperation is challenging.

In this paper, we propose a dynamic reward incentive mechanism. In this mechanism, the reward is determined by the system according to the proportion of agents with each strategy, i.e., when there are few cooperative agents, each cooperative agent receives a greater reward, but when there are more cooperative peers, each agent's reward is reduced. Thus, when all of the agents in the system are cooperative, no one receives a reward. The contributions of this work are as follows:

(1) A dynamic reward method is proposed that has a corrected reward function, and the proportion of each strategy is considered to determine the reward;

(2) Through our extensive theoretical analysis, we derive the evolutionary stable strategy under various scenarios;

(3) The qualitative analysis and the simulation prove that our proposed mechanism can promote cooperation effectively and reduce the cost of the system. 
The rest of the paper is structured as follows. In Section. 2, we briefly review related work on reward-based incentive mechanisms. In Section. B, we introduce our transaction game model and dynamic reward function. In Section. $\mathbb{\text { Q }}$, we use mathematical methods to predict the stable state of evolution. In Section. 5, the results of the simulation and performance analysis are presented. In Section. 6, our conclusions are presented.

\section{Related Work}

Providing incentives is one of the challenging issues in $\mathrm{P} 2 \mathrm{P}$ systems. The concept of incentive mechanisms is borrowed from economics and management and applied in computer science. The core thoughts of incentive mechanisms always target a specific behavior that is desirable in the system [7]. The essence is to help cooperative agents gain more benefit in order to encourage cooperation.

One of the most commonly-used approaches is to provide rewards and punishment. Agents who are willing to share can get a reward, while freeriders may be penalized. Although punishment is more effective than reward [10], sanctioning a penalty may result in "anti-social problems" [17]. Vice versa, rewards may lead to cooperation without harming agents' reputations or provoking any retaliation [18]. Thus a reward mechanism is healthier to achieve cooperation with less probability of any destructive results [1.9].

Generally, the source of rewards can be divided into two categories, i.e., peer rewards and institution rewards. For peer reward mechanisms, some volunteer peers act as "police" or "managers". They share their own resources and voluntarily reward contributing agents. However, such mechanisms require agents that are able to recognize and understand other agents' behaviors and that have the authority to reward (or punish) these agents $[20,21]$. It is difficult to achieve in a real system. Different from the peer reward approach, the institution reward mechanism uses a central server to track agents' behaviors and implement the reward mechanism. As in the institution reward mechanism, the rewards are given and managed by the central server, a mechanism that can be costly to the server. Especially, when a large number of cooperators must be rewarded, it can be either far too costly or ineffective. Thus, saving costs in the system under the premise of promoting cooperation is difficult to achieve. Ref [22] provides a novel insight in dynamic reward and punish mechanism by proposed "First carrot, then stick." The main idea is to reward or punish the minority peers in the 
system. However, this method must switch from reward to punishment, and properly setting the threshold switching mechanism is a great challenge. In addition, the settings for reward and punishment must be decided before implementing the mechanisms, and it is difficult to ensure the reward and punishment approach will always work well as the system evolves.

Peers in P2P systems are rational and strategic [2:3]. They participate in the system to maximize their benefits.Classic game theory, as a effective tool to model and explain rational behaviors under conflicts between individual and public interests, has been widely applied to the study and formal analysis of cooperation in $\mathrm{P} 2 \mathrm{P}$ networks [24, [25, [26]. In the classical game theoretic method, peers can get their best strategies by global knowledge, which requires great cognitive abilities (e.g., abilities of getting information of all global transactions and strong abilities of analyzing and recognizing). Such requirement is unfeasible in most cases. Due to the limited time for making decisions, lack of information or other unknown reasons, peers may have the probabilities making non-optimal decisions [27, 28]. This phenomena is called bound-rational. Moreover, classic game theory cannot characterize the evolutionary dynamics of peers strategies and how the strategies spread in the system. Recently, evolutionary game theory is used to analyze incentive mechanisms to overcome above drawbacks [2:9]. By leveraging evolutionary game theory, peers only need to learn and imitate more successful strategies of their neighbors. Such a simple imitative mechanism is easy to implement by peers limited knowledge to optimize their strategies, and more detailed insights into how the strategies spread in the network can be obtained. Generally, EGT [30] approaches consist of three major phases, i.e., interaction, evolution, and mutation [3I]. First, agents play games according to the rules specified by the system, and then each agent changes its strategy with the same rule. However, due to errors in the system or propagation issues, agents may change their strategies inappropriately, causing a small probability of mutation occurring. A general platform is required to evaluate incentive mechanisms. Zhao, et al. [32] provided a general framework for analyzing and evaluating three classic incentive mechanisms in well-mixed networks. In this paper, we focused on designing a dynamic reward mechanism based on EGT and test the effectiveness using the evaluation framework proposed by Zhao [32]. With this mechanism, a system effectively can encourage agents to cooperate, thereby reducing the cost associated with the system because no rewards are required when there are no free-riders to be eliminated. 


\section{System Model}

\subsection{System Description}

In this paper, we consider a mixed structure P2P system with a special peer which is used to maintain the system resource, user information and

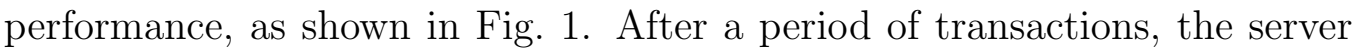
checks the transaction records of each agent and rewards those who are willing to share resources.

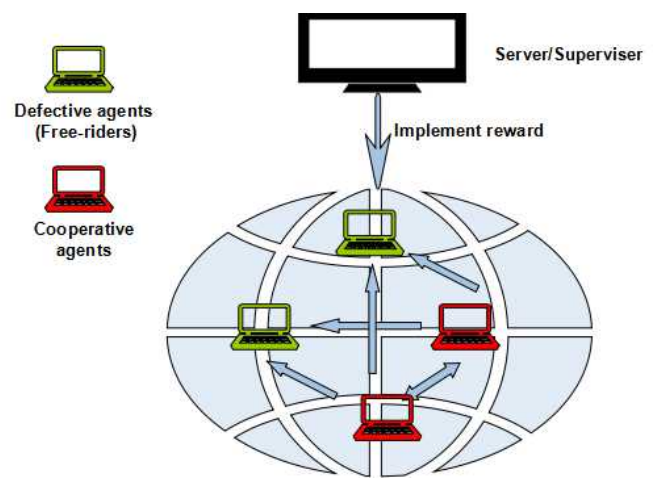

Figure 1: Mixed P2P structure with reward mechanism

A real autonomous system consists of $N$ different agents. Each agent $i$ is equally regarded as a service provider and a service requester in one transaction. A transaction may occur when two agents interact with each other, i.e., one agent requests a service and its opponent responds. Each time an agent provides a service, it suffers a cost, $\beta$. On the contrary, a requester gets a benefit, $\alpha$, when receiving a service. Participating in such systems may have other costs, such as the cost associated with acquiring a requester's transaction history. Therefore, the general average payoff of each agent, $P_{i}$, is related to the probability of its serving other agents $\left(\right.$ Prob $\left._{\text {serve }}\right)$, getting service $\left(\right.$ Prob $\left._{\text {get }}\right)$, and other additional costs (extra cost). The equation used to calculate payoff is shown as Eq. . For analysis simplicity, we set $\beta=1$.

$$
P_{i}=\alpha * \text { Prob }_{\text {get }}-\beta * \text { Prob }_{\text {serve }}-\text { extra_cost }
$$

Decentralized systems, such as the structure we use, can be classified into two scenarios: well-mixed and structured systems [34]. In a well-mixed population, an agent can interact with any other agent with equal probability in the system. Thus, we assume agents with same strategy share a common 
average payoff, and $P_{i}$ denotes the average payoff of every agent with strategy $i$. However, in a structured network, an agent can only interact with its direct neighbors, and the probability of an agent meeting another one agent is influenced by network structure. Therefore, even if agents have the same strategy, they may have different average payoffs. Thus, in a structured system, the parameter $P_{i}$ stands for the average payoff of agent $i$.

\subsection{Transaction Game}

A transaction game is described as a symmetric game between two agents. In brief, we consider the three most common strategies, i.e., C, R, and D. The behaviors of those who use these strategies are depicted as follows (Fig.2]): C users (cooperators) always grant a service without considering the history of transactions; D users (defectors) always deny a service; R users always are called reciprocators, because they grant services to cooperators and reciprocators and deny defectors requests [35].According to the definition of the game model, we write the payoff matrix A as Eq. 2. Each item $a_{i j}$ of this matrix symbolizes the payoff of one agent with strategy $S_{i}$ when it meets another agent with strategy $S_{j}$. Take the item $a_{21}$ for instance: the value is the payoff of a reciprocator when it meets a cooperator. If a reciprocator meets a cooperator, it will always grant a service to the cooperator and get a service from the cooperator. Thus it can obtain a benefit $\alpha-1$. However, to know the opponent's strategy, the reciprocator has to search the server for information. So it has to bear an extra cost $C_{R}$. Therefore, the total payoff of a reciprocator in this transaction is $\alpha-1-C_{R}$. Since both cooperators and reciprocators provide services, we call them cooperative agents, and defectors are called defective agents.

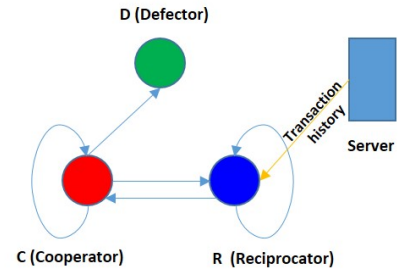

Figure 2: Description of the transaction game 


$$
A=\begin{gathered}
C \\
R \\
D
\end{gathered}\left(\begin{array}{ccc}
C & R & D \\
\alpha-1 & \alpha-1 & -1 \\
\alpha-1-C_{R} & \alpha-1-C_{R} & -C_{R} \\
\alpha & 0 & 0
\end{array}\right)
$$

\subsection{Reward Mechanism}

The existence of P2P systems strongly relies on the voluntary of agents. However, sharing is costly. To encourage sharing behaviors, a reward mechanism is needed to help cooperative agents cover the cost of sharing resources [14]. Rewards can be modeled as quantitative changes in parameters associated with an agent[7]. In this paper, we adopt an institutional reward mechanis$\mathrm{m}$ : the reward is given by the central server to those cooperative agents $(\mathrm{C}$ and R) for their willingness to help other agents. Each time an agent shares resources with another agent, the central server gives the agent a reward in praise of voluntary behavior and help covering sharing costs. As the reward is given by the server, the server has to bear the cost. Thus, to alleviate the cost of giving rewards, we designed a dynamic reward-based incentive. Bearing this reward mechanism, the payoff matrix $(A)$ can be modified as Eq. 3. As mentioned earlier, the system must bear a cost, $C_{S}$, the value of which can be calculated as Eq. $⿴$, where $N_{C}$ and $N_{R}$ are the number of cooperators and reciprocators in the system, respectively.

$$
\begin{gathered}
A=\begin{array}{c}
C \\
C \\
R \\
D
\end{array}\left(\begin{array}{ccc}
\alpha-1+r d & \alpha-1+r d & -1+r d \\
\alpha-1+r d-C_{R} & \alpha-1+r d-C_{R} & r d-C_{R} \\
\alpha & 0 & 0
\end{array}\right) \\
C_{S_{F}}=r d *\left(N_{C}+N_{R}\right)
\end{gathered}
$$

\subsection{Strategy Dynamics}

Updating the strategy is a critical process in the evolution of the population. Those agents who have better payoff are favored by the system and have higher probability of surviving in the following transactions. In this paper, we used the most common pairwise comparison update rule, i.e., the Fermi update [36, 37, 38, 3.9, 40]]. After each round, an agent $i$ randomly selects another agent $j$ in the system (a neighbor in a structured network). Player 
i will learn to change its strategy to player $j$ s strategy with and calculate the probability of changing its strategy to that of agent $j$ by using the Fermi function (Eq. 回). The parameter $w$ is the selection intensity. The larger $w$ becomes, the faster the evolution proceeds. To make sure the system evolves smoothly, we set $w=10$.

$$
p_{i \rightarrow j}=\frac{1}{1+\exp \left[w\left(p_{i}-p_{j}\right)\right]}
$$

\section{Theoretical Analysis}

Replicator dynamics [41] are the most common mathematical tools that are used to analyze the evolution of the population. A core concept is fitness, which represents the capability an agent to survive in the system. The evolution of the population can be expressed by a differential equation (Eq. G):

$$
\dot{x}=x_{i}\left(f_{i}-\phi\right), \text { where } \sum_{i} x_{i}=1
$$

In this equation, $f_{i}$ is the fitness of strategy, $S_{i}$, and $f_{i}=\sum_{i} x_{i} a_{i j}$. The parameter $\phi$ is the average fitness, and $\phi=\sum_{i} x_{i} f_{i}$. If the fitness of strategy, $S_{i}, f_{i}$, is larger than the system average fitness, $\phi\left(f_{i}>\phi\right)$, the population of $S_{i}$ will grow and survive in the long run. On the contrary, the worse strategy will decrease and finally disappear. Thus, to analyze the evolutionary stable strategy (ESS), we need only to compare the fitness of each strategy and find the threshold of each result. The notations used in this paper are listed in Table. . 
Table 1: Definition of notations

\begin{tabular}{ll}
\hline Symbol & Definition \\
\hline$N$ & number of agents in the system \\
$\alpha$ & benefit when getting a service \\
$\beta$ & cost when providing a service \\
$C_{R}$ & cost for reciprocators to seek for information \\
$r d$ & reward given to cooperative agents \\
$i$ & an agent in the system \\
$S_{i}$ & strategy $i$ \\
$x_{i}$ & the proportion of agents with strategy $S_{i}$ \\
$P_{i}$ & the payoff/fitness of strategy $S_{i}$ \\
$w$ & selection intensity \\
$p_{i \rightarrow j}$ & the probability that agent $i$ will use $j$ s strategy \\
\hline
\end{tabular}

\subsection{Well-mixed Population}

In a well-mixed population, each agent can interact with any other agent with equal probability. So, the average payoff is directly related to the proportion of each strategy. Let $x_{0}, x_{1}$, and $x_{2}$ denote the proportion of $\mathrm{C}, \mathrm{R}$, and $\mathrm{D}$ agents, respectively. Then, the average payoff of each strategy can be written as Eq. [:

$$
\begin{aligned}
& P_{0}=\alpha\left(x_{0}+x_{1}\right)-1 \\
& P_{1}=\alpha\left(x_{0}+x_{1}\right)-\left(x_{0}+x_{1}\right)-C_{R} \\
& P_{2}=\alpha x_{0}
\end{aligned}
$$

\subsubsection{No Reward}

Without considering the cost reciprocators seeking information $\left(C_{R}=0\right)$, we can derive the respective payoff as follows:

$$
\begin{aligned}
& P_{0}=\alpha\left(x_{0}+x_{1}\right)-1 \\
& P_{1}=\alpha\left(x_{0}+x_{1}\right)-\left(x_{0}+x_{1}\right) \\
& P_{2}=\alpha x_{0}
\end{aligned}
$$

Based on the above expressions, we have the following important observations: 
(1) As the summation of $x_{0}+x_{1}$ is always less than or equal to 1 , there will always be $P_{0} \leq P_{1}$. Thus, to eliminate defectors, we need $P_{1}>P_{2}$ or $P_{0}>P_{2}$. If $P_{1}>P_{2}$, we have $\alpha>\frac{x_{0}+x_{1}}{x_{0}}$; if $P_{0}>P_{2}$, we have $\alpha>\frac{1}{x_{1}}$.

(2) When $\alpha<\frac{x_{0}+x_{1}}{x_{1}}$, we have $P_{2}>P_{1}>P_{0}$. In other words, defectors have the best fitness, therefore defecting is the best strategy until $\alpha=\frac{x_{0}+x_{1}}{x_{1}}$.

(3) When $\frac{x_{0}+x_{1}}{x_{1}}<\alpha<\frac{1}{x_{1}}$, we have $P_{1}>P_{2}>P_{0}$, cooperators will evolve to defectors and reciprocators. When there are only defectors and reciprocators, if $\alpha>1$, the ESS is R; When $\alpha=1$, neutral drift occurs between reciprocators and defectors.

(4) If $\alpha>\frac{1}{x_{1}}$, we have $P_{1}>P_{2}>P 2$, therefore cooperators and reciprocators co-exist at stable status.

In general, when $C_{R}=0$, for any $\alpha>1, \mathrm{R}$ is the only ESS.

However, in a real system, to get information, an agent must incur a certain cost. When $C_{R}>0, p_{1}=\alpha\left(x_{0}+x_{1}\right)-\left(x_{0}+x_{1}\right)-C_{R}$, and defectors can be wiped out only if $C_{R}<(\alpha-1) x_{1}-x_{0}$. When there are only $\mathrm{D}$ and $\mathrm{R}, x_{0}=0$, the inequation becomes $C_{R}<(\alpha-1) x_{1}$.

\subsubsection{Fixed and dynamic reward}

By involving the reward mechanism, cooperator and reciprocators can get an additional benefit from the system. Thus, the payoff equations can be modified as follows:

$$
\begin{aligned}
& P_{0}=\alpha\left(x_{0}+x_{1}\right)-1+r d \\
& P_{1}=\alpha\left(x_{0}+x_{1}\right)-\left(x_{0}+x_{1}\right)-C_{R}+r d \\
& P_{2}=\alpha x_{0}
\end{aligned}
$$

In this case $r d$ is the reward value set by the system administrator before the system starts. To eliminate side effect of defectors, we should ensure cooperative agents get best payoff. Thus, using the same method as in Sec. 4.I. I, we can get the following results:

(1) If $P_{0}>P_{1}>P_{2}$, we have $C_{R}>x_{2}$ and $r d>C_{R}+x_{0}+(1-\alpha) x_{1}$. Therefore, defectors will learn to behave cooperatively. When $r d=$ $C_{R}+x_{0}+(1-\alpha) x_{1}$, neutral drift occurs between reciprocators and 
defectors. However, these two strategies both evolve to cooperating strategy. Thus cooperators will dominate until the inequation isn't satisfied.

(2) If $P_{1}>P_{0}>P_{2}$, we have $C_{R}<x_{2}$ and $r d>1-\alpha x_{1}$. When $r d=1-$ $\alpha x_{1}$, neutral drift occurs between cooperators and defectors. However, these two strategies both evolve to the reciprocative strategy. As $x_{1}$ increases, we'll always have $r d>1-\alpha x_{1}$. Thus, R strategy will be the ESS.

(3) When neither of the former two cases are fulfilled, D will dominate until one of the first two cases is satisfied and it returns to one of the first cases.

From the aforementioned fixed reward, we know that, if $r d$ always satisfies the first two cases, we can make sure the defectors are defeated. However, no matter $P_{0}>P_{1}$ or $P_{0}<P_{1}$, if we can ensure that $P_{1}>P_{2}$, the defectors will involve to reciproctors, then we can get full cooperative agents. Thus, to reduce the cost of the system and ensure the effectiveness of the incentive, we use a dynamic reward function. Each time the system incurs a reward, it calculates the real-time reward, as shown in Eq. $\nabla$. So the calculation of the systems cost can be modified as Eq. 9, where $N_{0}$ and $N_{1}$ are the number of cooperators and reciprocators, respectively, and $\delta>0$.

$$
\begin{gathered}
r d\left(x_{0}, x_{1}\right)=\max \left(1-\alpha x_{1}+\delta, 0\right) \\
C_{S_{D}}=r d\left(x_{0}, x_{1}\right) *\left(N_{0}+N_{1}\right)
\end{gathered}
$$

In the scenario with dynamic reward, the system cost becomes a function of the proportion of defectors (Eq. Q $⿴ 囗 ⿱ 一 一)$ ). Thus, the cost difference between a dynamic and a fixed reward $\left(\Delta=C_{S_{D}}-C_{S_{F}}\right)$ can be expressed as Eq.

$$
\Delta=\left[\left(x_{0}-x_{0}^{0}\right)+(1-\alpha)\left(x_{1}-x_{1}^{0}\right)\right]\left(N_{0}+N_{1}\right)
$$

As analyzed before, when $C_{R}>x_{2}, \mathrm{C}$ agents evolve to $\mathrm{R}$ and $\mathrm{D}$ agents, and $\mathrm{D}$ agents evolve to $\mathrm{R}$ agents. So, $x_{0}$ decreases, and $x_{1}$ increases. So the inequation is $\Delta<0$, which means that using a dynamic reward can reduce the cost of the system. When $C_{R} \leq x_{2}$, the proportion of reciprocators keeps increasing, thus $\Delta$ will always be less than 0 . Therefore, in general, with 


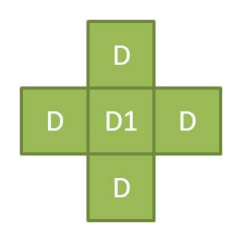

(a) $P_{D 1}=0$

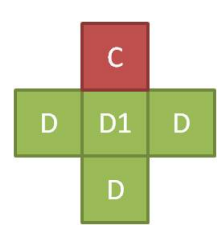

(b) $P_{D 1}=\frac{\alpha}{4}$

Figure 3: Example of a defector's location and payoff

dynamic reward, the cost of the system for each agent can be reduced, and the the total payment for rewards also can be reduced in the long run.

\subsection{Structured Networks}

Different from a well-mixed population, in a structured network, agents can only interact with their neighbors. Thus, the location of an agent can influence the interaction among agents. For instance, a defector that is surrounded by four defectors can get no payoff (Fig. 3(a)). However, a defector surrounded by one cooperator and three agents with other strategies may get an $\alpha / 4$ average payoff (Fig. 3(b)) . Therefore, the correlation of two adjacent agents strategies should be taken into account.

To analyze the evolution on a structured network, we used replicator dynamics on graph [42] and considered the pairwise comparison update, such as the Fermi update rule. With the influence of the networks structure, for a network with average degree $k>2$, the dynamic equation becomes $\dot{x}_{i}=x_{i}\left(f_{i}+g_{i}-\phi\right)$. For pairwise comparison updating, such as the Fermi update rule, the parameters in this replicator equation are as follows:

$$
\begin{aligned}
f_{i} & =\sum_{j} x_{j} a_{i j} \\
b_{i j} & =\frac{a_{i i}+a_{i j}-a_{j i}-a_{j j}}{k-2} \\
g_{i} & =\sum_{j} x_{j} b_{i j} \\
\phi & =\sum_{i} x_{i}\left(f_{i}+g_{i}\right)=\sum_{i} x_{i} f_{i}
\end{aligned}
$$


The term of $b_{i j}$ describes local competition between strategy $S_{i}$ and $S_{j}$, and $g_{i}$ characterizes the local competition among strategies. Note that the calculation of the value of $b_{i j}$ involves the four items from the symmetric payoff matrix, so we can derive $b_{i j}=b_{j i}$. Thus $\sum_{i=1}^{n} g_{i}=0$ and $\phi=\sum_{i} x_{i}\left(f_{i}+\right.$ $\left.g_{i}\right)=\sum_{i} x_{i} f_{i}$, where $\sum x_{i}=1$. Absorbing this method, we get a new matrix $B$ (Eq. $\square$ ). Then, the new payoff matrix $C$ can be modified as each item $c_{i j}=a_{i j}+b_{i j}$ (Eq. ए2).

$$
\begin{aligned}
& B=\begin{array}{c}
C \\
C \\
R \\
D
\end{array}\left(\begin{array}{ccc}
C & R & D \\
0 & \frac{2 C_{R}}{k-2} & \frac{2 r d-2}{k-2} \\
-\frac{2 C_{R}}{k-2} & 0 & \frac{\alpha-1+2 r d-2 C_{R}}{k-2} \\
-\frac{2 r d-2}{k-2} & -\frac{\alpha-1+2 r d-2 C_{R}}{k-2} & 0
\end{array}\right) \\
& C=\begin{array}{c}
C \\
C \\
R \\
D
\end{array}\left(\begin{array}{ccc}
\alpha-1+r d & \alpha-1+r d+\frac{2 C_{R}}{k-2} & \frac{k(r d-1)}{k-2} \\
\alpha-1+r d-\frac{k C_{R}}{k-2} & \alpha-1+r d-C_{R} & \frac{\alpha-1+k\left(r d-C_{R}\right)}{k-2} \\
\alpha-\frac{2 r d-2}{k-2} & -\frac{\alpha-1+2 r d-2 C_{R}}{k-2} & 0
\end{array}\right)
\end{aligned}
$$

For the convenience of further analysis, we define a new variable $h_{i}=f_{i}+g_{i}$, and then we have the average fitness of three strategies (Eq. [13):

$$
\begin{aligned}
h_{0} & =\frac{\left(k-2+x_{2}\right) r d+2 C_{R}-k x_{2}}{k-2}+\left(1-x_{2}\right)(\alpha-1) \\
h_{1} & =\left(1+\frac{2 x_{2}}{k-2}\right)(\alpha-1+r d)-\frac{k-2 x_{1}}{k-2} C_{R} \\
h_{2} & =x_{0}\left(\alpha-\frac{2 r d-2}{k-2}\right)-x_{1} \frac{\alpha-1+2 r d-2 C_{R}}{k-2}
\end{aligned}
$$




\subsubsection{No reward}

First, we consider the condition with no information cost or reward. If we let $C_{R}=0$ and $r d=0$, then we have:

$$
\begin{aligned}
h_{0} & =\left(1-x_{2}\right)(\alpha-1)-\frac{k x_{2}}{k-2} \\
h_{1} & =(\alpha-1)\left(1+\frac{2 x_{2}}{k-2}\right) \\
h_{2} & =x_{0}\left(\alpha+\frac{2}{k-2}\right)-x_{1} \frac{\alpha-1}{k-2}
\end{aligned}
$$

Based on the above expressions, we have the following important observations:

(1) When $h_{0}-h_{1}=-x_{2} \frac{k \alpha}{k-2} \leq 0$, the reciprocators will always have better performance than the cooperators. When $x_{2}=0$, neutral drift occurs between the cooperators and reciprocators, and they will co-exist.

(2) When $\alpha>\frac{k+(k-4) x_{2}-x_{1}}{(k-1) x_{1}}$, we have $h_{1}>h_{0}>h_{2}$. Thus, defectors will learn to behave cooperatively. As $x_{1}$ increases and $x_{2}$ decreases, the value of $\frac{k+(k-4) x_{2}-x_{1}}{(k-1) x_{1}}$ decreases, the inequation will always be satisfied. Therefore, defectors will be conquered in the end.

(3) When $\alpha<\frac{1-x_{1}}{1-x_{0}-\frac{3}{k} x_{1}}$, we have $h_{0}<h_{1}<h_{2}$. Thus, defecting is the best strategy and dominate the system.

(4) When $\alpha>\frac{1-x_{1}}{1-x_{0}-\frac{3}{k} x_{1}}$, we have $h_{1}>h_{2}$. Thus defectors will evolve to reciprocators. However, if we cannot fulfil the inequation in Case (2), cooperators will evolve and become defectors and reciprocators. In this case, when there are only defectors and reciprocators, $\mathrm{R}$ adopters will dominate until $\alpha=\frac{1-x_{1}}{1-\frac{3}{k} x_{1}}$ and then neutral drift will occur between them and they will co-exist continuously. 
When we consider the cost seeking information, $C_{R}>0$ and $r d=0$, we have:

$$
\begin{aligned}
h_{0} & =\frac{2 C_{R}-k x_{2}}{k-2}+\left(1-x_{2}\right)(\alpha-1) \\
h_{1} & =\frac{2 x_{2}+k-2}{k-2}(\alpha-1)-\frac{k-2 x_{1}}{k-2} C_{R} \\
h_{2} & =x_{0}\left(\alpha+\frac{2}{k-2}\right)-x_{1} \frac{\alpha-1-2 C_{R}}{k-2}
\end{aligned}
$$

In this case, we get get results as follows:

A When $C_{R}>\frac{k x_{2}}{k-2-2 x_{1}} \alpha, h_{0}>h_{1}$;

B when $C_{R}>\frac{x_{1}+(k-1)\left(2 x_{2}-\alpha\right)+k-2}{2\left(1+x_{1}\right)}, h_{0}>h_{2}$;

C when $C_{R}<\frac{(k-4) x_{0}-x_{1}}{k+2 x_{1}} \alpha-\frac{k+x_{1}+2 x_{2}-2}{k+2 x_{1}}, h_{1}>h_{2}$.

From the above cases, we can derive the following: when the status of the system satisfies case A and B at the same time, we can be sure that the system will evolve to the status of being fully comprised of cooperative agents.

\subsubsection{Fixed and dynamic rewards}

Under the influence of reward mechanism, we used the fitness functions as shown in Eq. 13 and we solved the similar inequations. The results showed that:

(1) When $r d>C_{R}+\frac{(k-1) x_{0}-(k-1)(\alpha-1) x_{1}-(\alpha-1) x_{2}}{k}$, R agents will rule the system

(2) When there are only R and D users, if $r d>C_{R}-\frac{(k-1)(\alpha-1) x_{1}+(\alpha-1) x_{2}}{k}$, $\mathrm{R}$ strategy is the ESS.

(3) When only $\mathrm{R}$ and $\mathrm{C}$ agents in the system. $h_{0}-h_{1}=\frac{k}{k-2} C_{R}>0$. Thus, when these two strategies co-exist, C strategy is the only ESS.

Similarly, when the reward is dynamic, if the value of the reward is slightly larger than the threshold, the system effectively can become cooperative, and the cost of the system will be reduced. In this scenario, we set the dynamic reward function as Eq. [4, $\vec{x}$ symbolizes the fraction of each strategy.

$$
r d\left(\vec{x}, C_{R}\right)=C_{R}-\frac{(k-1)(\alpha-1) x_{1}+(\alpha-1) x_{2}}{k}+\delta
$$




\section{Performance Evaluation}

The performance evaluation results are presented in this section. Initially, we investigated and compared the performance of the proposed incentive mechanism with dynamic rewards and fixed rewards. Unless there was a special need, we used the parameters in Table. 2 .

Table 2: Parameters

\begin{tabular}{lll}
\hline Parameter & Definition & value \\
\hline$N$ & number of agents in the system & 10,000 \\
$\beta$ & cost when providing a service & 1 \\
$\left(x_{0}, x_{1}, x_{2}\right)$ & initial proportion of each strategy & $(0.3,0.2,0.5)$ \\
$w$ & selection intensity & 10 \\
$<k>$ & average degree of structured networks & 4 \\
\hline
\end{tabular}

Our simulation was divided into Monte Carlo steps. As described in Alg. 1 , in each step, an agent plays the transaction game with its all neighbors, excluding itself (with all agents in a well-mixed population). Then, each agent simultaneously calculates its payoff and adopts a new strategy according to the Fermi update. In addition, a real system architecture is always structured, and we also simulated a structured network by using a square lattice and captured the snapshots along with the evolution of the system.

\subsection{Effectiveness of incentives in a well-mixed population}

In a well-mixed population, each agent can interact with any other agent in the system. Figs. 田-6 show the evolution of strategies in well-mixed population under different scenarios.

As we analyzed in Sec. A.I, when there is no reward, if $\alpha$ is smaller than the threshold, the system will be filled with defectors, but, if there are rewards, the system will be filled with reciprocators. However, cooperators always have less payoff than reciprocators and defectors, so they converge to $\mathrm{D}$ and $\mathrm{R}$ and disappear in the system. When there are only $\mathrm{D}$ and $\mathrm{R}$, $=1$ is the systems threshold. When $\alpha<1$, defectors rule; when $\alpha>1$, reciprocators rule; and, when $=1$, neutral drift occurs between defectors and reciprocators (Fig. 团).

In a real system, when an agent needs some information, it must incur a cost (e.g., bandwidth and CPU cycle [43]). Thus, it is necessary to consider 


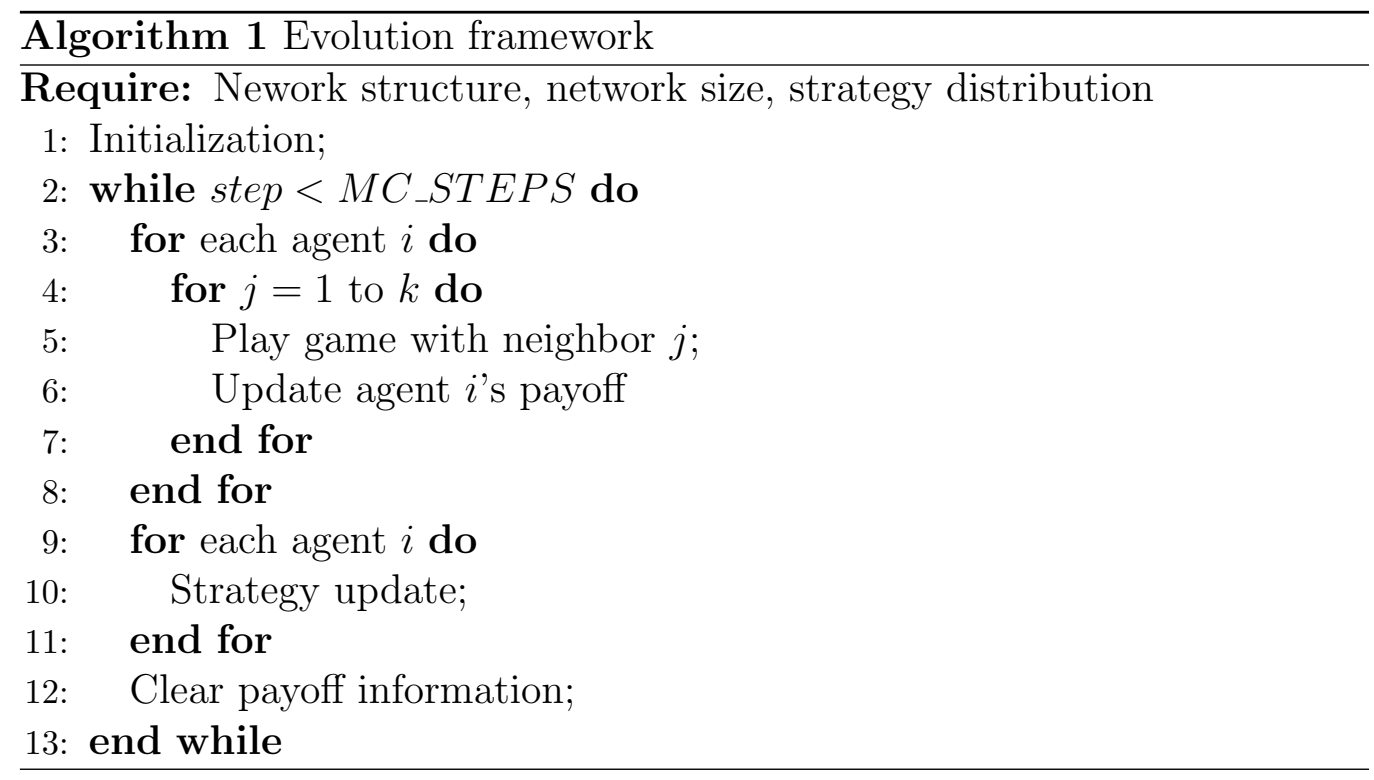

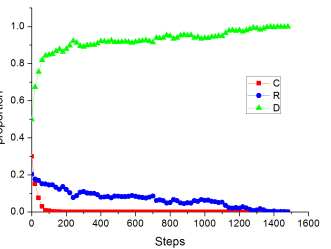

(a) Defectors Rule

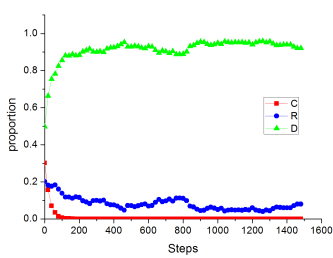

(b) Neutral Drift

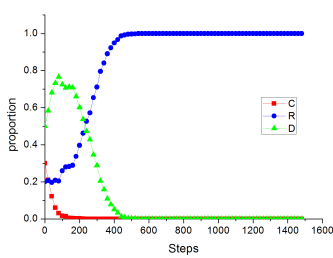

(c) Replicators Rule

Figure 4: Evolution with different $\alpha$ and $0 \operatorname{cost}(\mathrm{a}) \alpha=0.5$ (b) $\alpha=1$ (c) $\alpha=1.5$ 


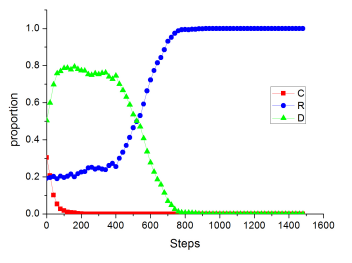

(a) $C_{R}<(\alpha-1) x_{1}$

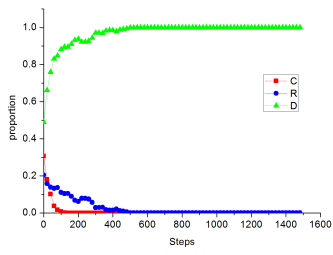

(b) $C_{R}>(\alpha-1) x_{1}$

Figure 5: Evolution with different cost, $\alpha=1.5$

Initial proportion of each strategy $\left(x_{0}, x_{1}, x_{2}\right)=(0.3,0.2,0.5)$ (a) $C_{R}=0.075$ (b) $C_{R}=$ 0.15

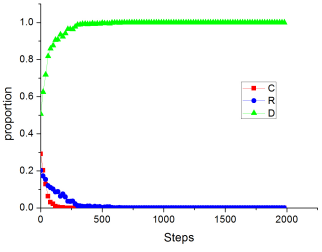

(a) $r d=0.1$

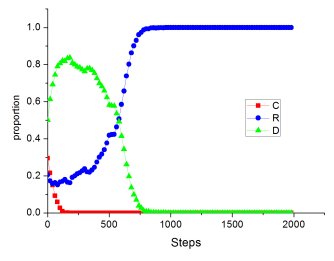

(b) $r d=0.25$

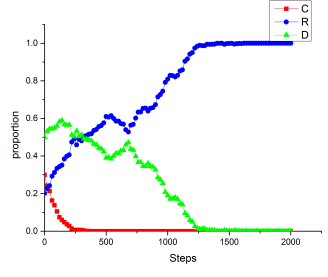

(c) Dynamic Reward $(\delta=$ $0.05)$

Figure 6: Evolution with different reward, $\alpha=1.5, C_{R}=0.3$ Initial proportion of each strategy $\left(x_{0}, x_{1}, x_{2}\right)=(0.3,0.2,0.5)$

Even though the reward mechanism can promote cooperation, the system will incur extra costs. As shown in Fig. I, for a fixed reward system, when the system is full of cooperative agents, all of them must be paid. The accumulative cost keeps increasing as more agents evolve and become more cooperative. However, when we consider dynamic reward, since there are 
fewer and fewer defectors, the reward for each agent decreases. Thus, the cumulative cost will decrease in the long run.

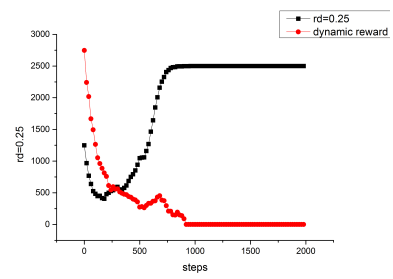

Figure 7: System cost with reward $(\delta=0.05)$

\subsection{Effectiveness of incentives in a structured network}

In a real system, a well-mixed population rarely exists. To make the model more realistic, we tested our model on a square lattice, a commonly used network structure when analyzing the evolution of strategies. Initially, each agent processes a vertice of a $100 * 100$ square lattice and assigned a strategy with the distribution $\left(x_{0}, x_{1}, x_{2}\right)=(0.3,0.2,0.5)$, which was the same as the distribution in a well-mixed population. In this circumstance, each agent plays a transaction game with each neighbor once (excluding itself). To understand the influence of the networks structure, we captured the curves of evolution and the snapshots of each step.

First, we investigated the simplest scenario, i.e., without information cost and reward (Fig. 8). Similar to the results in a well-mixed population, =1 is the evolution threshold. But, due to the correlations of agents, the speed of evolution is faster.

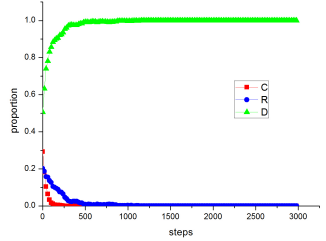

(a) Defectors Rule

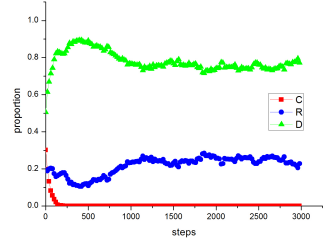

(b) Neutral Drift

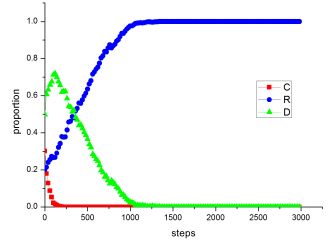

(c) Replicators Rule

Figure 8: Evolution with different $\alpha$ and 0 cost on a square lattice (a) $\alpha=0.5$ (b) $\alpha=1$ (c) $\alpha=1.5$ 
To see more clearly how the correlation of strategies influences the systems dynamics, we captured the evolution snapshot (Fig. 91). In the first few steps, agents tend to form small clusters with the same strategy. Without considering $C_{R}$ and reward, under three different values of, cooperators always have the worst payoff. So, unless they are surrounded by agents with the same strategy, they will change their strategy, C, to the other two strategies [Figs.9(b), 9(f)],9(j)]. However, when there are only reciprocators and defectors, the results differ. When $\alpha<1$, a reciprocator, at most, can get zero payoff when surrounded by four defectors. Even if theres only one reciprocator links with it, it gets a negative payoff. So, the cluster of reciprocators narrows until it disappears [Figs. $9(\mathrm{~g})$, 9(h)]. Opposite from this scenario, when $\alpha>1$, payoff of a reciprocator is greater than or equal to 0 , but defectors can only get 0 payoff, as they cannot get service from defectors or reciprocators [Figs.9(k), 9(1)]. When $\alpha=1$, they all get 0 payoff, so neutral drift occurs, and the system will maintain its stable state.

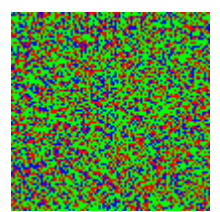

(a) Initial

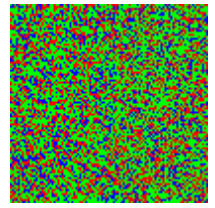

(e) Initial

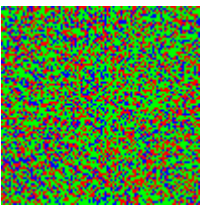

(i) Initial

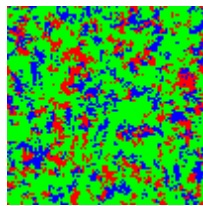

(b) $\mathrm{step}=20$

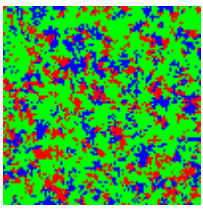

(f) $\mathrm{step}=20$

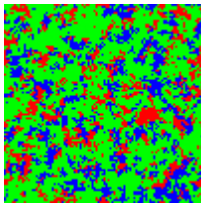

(j) $\mathrm{step}=20$

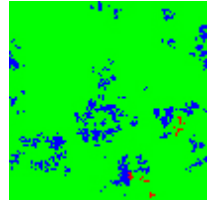

(c) $\mathrm{step}=200$ step $=1200$
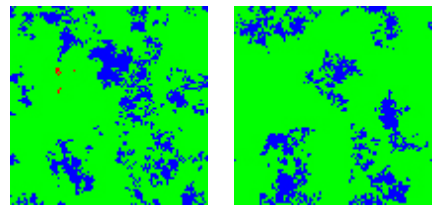

(g) $s t e p=200$

(h) $\mathrm{step}=700$
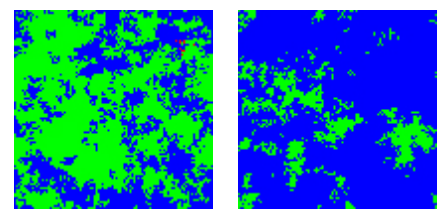

(k) $\mathrm{step}=200$

(l) $\mathrm{step}=1500$

Figure 9: Snapshot of the evolution strategies top to bottom: $\alpha=0.5,1,1.5$ red nodes: cooperators, green nodes: defectors, blue nodes: reciprocators 
When we consider the cost of seeking information $\left(C_{R}>0\right)$, the payoff of reciprocators changes. If $C_{R}$ is small, reciprocators can still conquer defectors as they may still get a positive payoff when there are only $\mathrm{R}$ and $\mathrm{D}$ agents (Fig.10). But when the cost is larger than the threshold we analyzed in Sec. 4.2, reciprocators can no longer exist.

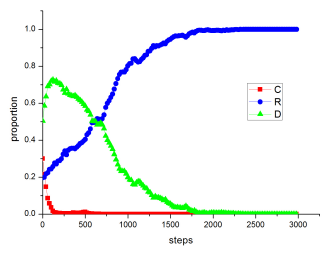

(a) $C_{R}=0.1$

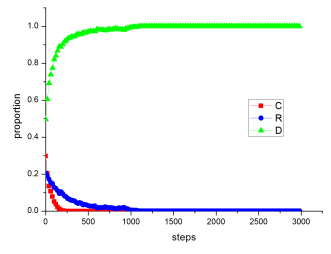

(b) $C_{R}=0.3$

Figure 10: Evolution with different cost, $\alpha=1.5$

Initial proportion of each strategy $\left(x_{0}, x_{1}, x_{2}\right)=(0.3,0.2,0.5)$

(a) $C_{R}=0.1$ (b) $C_{R}=0.3$

A cost for knowing more information is mandatory in most systems. To encourage agents to be more cooperative, we still consider the reward mechanism. Similar with well-mixed population, when the value of the reward was less than the threshold, the reward provides very little incentive, and it can slightly decrease the speed of the defectors invasion (Fig.11(a)). When the reward is larger than the threshold, the system will converge to full cooperative agents (Fig.11(b)). As the system is dynamic, a proportion of each strategy changes all the time, and we consider the dynamic reward. The rewards value is slightly larger than the threshold, and, when the system is full of cooperative agents, we set $r d=0$. Thus, the dynamic reward can effectively promote cooperation as well. 


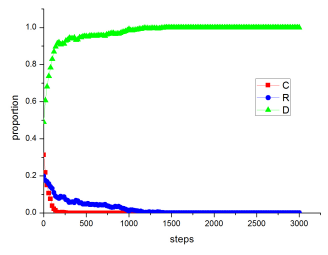

(a) Defectors Rule

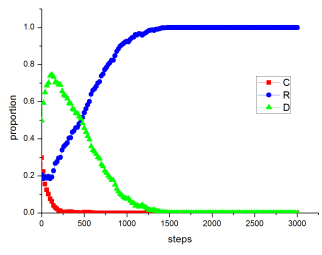

(b) Neutral Drift

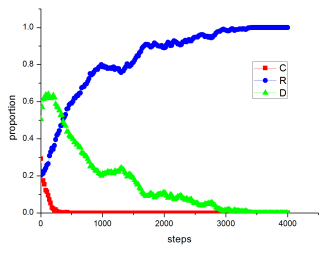

(c) Replicators Rule

Figure 11: Evolution with different reward $(r d)$ on a square lattice (a) $r d=0.08$ (b) $r d=0.3$ (c) Dynamic reward

The main purpose of using a dynamic reward is to reduce the cost of the system. So, we compare the overall system cost in each step (Fig. [22). In a fixed reward system, as the number of cooperative agents keeps increasing, the systems cost also increases. When the system is full of cooperative agents, the cost will stay at the peak in all following rounds. But when we consider dynamic reward, the system cost will decrease as with less defectors, a small reward will help defeat defectors. When defectors disappear, the system no longer needs rewarding agents, and the cost of the system can be reduced.

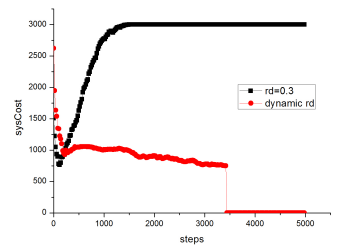

Figure 12: System cost with reward on a square lattice

\section{Conclusions}

To solve the free-riding problem in $\mathrm{P} 2 \mathrm{P}$ systems, we introduced a dynamic reward mechanism into classical incentive mechanisms. Through theoretical analysis, we found the optimal system settings to encourage agents to cooperate, and we compared the effectiveness of each scenario. In addition, we theoretically and qualitatively proved that the cost of the system can be reduced with a dynamic reward. Through numerous simulations from different aspects, we tested and confirmed the results that were obtained from the 
analysis. A relatively large reward may lead the system to be fully cooperative. However, using dynamic reward can get the same effect, but dynamic reward may reduce the cost of the system in the long run. With the growing use of new, decentralized, and self-organized methods of computing and sensing, these systems are facing similar problems concerning cooperation. Our paper provides a simple and effective way to implement rewards in a real system.

However, for further studies, we may consider the dynamic of networks as well, when agents join and leave the system at various times. The coevolution of strategies and network topology will be our research direction in the following studies.

\section{Acknowledgement}

This paper is supported by the National Science Foundation of China under grant No. 61272173, 61403059 and 61572095.

\section{References}

[1] eDonkey, http://www.verycd.com/

[2] PPLive, http://www.pptv.com

[3] PPStream., http://www.ppstream.com

[4] Wang Y, Nakao A, Ma J. (2010) Psychological research and application in autonomous networks and systems: A new interesting field. 2010 International Conference on Intelligent Computing and Integrated Systems (ICISS), 568-572

[5] Adar, Eytan and Huberman, Bernardo A. (2000) Free riding on Gnutella. First Monday, 5(10)

[6] Haddi, F. L., \& Benchaba, M. (2015). A survey of incentive mechanism$\mathrm{s}$ in static and mobile P2P systems. Journal of Network and Computer Applications, 58, 108118.

[7] O. Scekic, H. L. Truong, S. Dustdar (2013) Incentives and rewarding in social computing. Communications of the ACM, 56(6):72-82. 
[8] Karl Sigmund. (2007) Punish or perish? retaliation and collaboration among humans. Trends in Ecology and Evolution, 22(11):593-600.

[9] Daniel, B., Mulder, L. B., \& Lange, P. A. M. V. (2011) Reward, punishment, and cooperation: a meta-analysis. Psychological Bulletin, 137(4), $594-615$.

[10] Yufeng Wang, Athanasios V. Vasilakos, \& Jianhua Ma. (2011) Punishment or reward: it is a problem in anonymous, dynamic and autonomous networking environments. In Ubiquitous Intelligence and Computing, 197208.

[11] Mahmoud, S., Villatoro, D., Keppens, J., \& Luck, M. (2012) Optimised Reputation-Based Adaptive Punishment for Limited Observability. Proceedings of the 2012 IEEE Sixth International Conference on Self-Adaptive and Self-Organizing Systems, 129-138.

[12] Brun, O., El-Azouzi, R., Prabhu, B., \& Seregina, T. (2014) Modeling rewards and incentive mechanisms for Delay Tolerant Networks. 2014 12th International Symposium on Modeling and Optimization in Mobile, Ad Hoc, and Wireless Networks (WiOpt),233-240.

[13] Wu, T. Y., Lee, W. T., Guizani, N., \& Wang, T. M. (2014) Incentive mechanism for P2P file sharing based on social network and game theory. Journal of Network \& Computer Applications, 41(3), 47-55.

[14] Wu, W., Ma, R. T., \& Lui, J. (2014) Distributed caching via rewarding: An incentive scheme design in P2P-VoD systems. IEEE Transactions on Parallel and Distributed Systems, 25(3), 612-621.

[15] Sigmund K. , Hauert C. \& Nowak, M. A. (2001) Reward and punishment. Proceedings of the National Academy of Sciences of the United States of America, 98(19), 284-290.

[16] Zhang, K., Antonopoulos, N., \& Mahmood, Z. (2009) A Review of Incentive Mechanism in Peer-to-Peer Systems. International Conference on Advances in P2P Systems, 45-50.

[17] Benedikt, H., Christian, T., \& Simon, G. (2008) Antisocial punishment across societies. Science, 319(5868), 1362-1367. 
[18] Manfred, M., Dirk, S., \& Hans-Jrgen, K. (2002) Reputation helps solve the 'tragedy of the commons'. Nature, 415(6870), 424-426.

[19] Rand, D. G., \& Nowak, M. A. (2011) The evolution of antisocial punishment in optional public goods games. Nature Communications, 2(1), $73-86$.

[20] Sasaki T. (2014) The evolution of cooperation through institutional incentives and optional participation. Dynamic Games \& Applications, 4(3), $345-362$.

[21] Casari, M., \& Luini, L. (2009) Cooperation under alternative punishment institutions: an experiment. Journal of Economic Behavior \& Organization, 71(2), 273-282.

[22] Xiaojie Chen, Tatsuya Sasaki, ke Brnnstrm, Ulf Dieckmann. (2015) First carrot, then stick: how the adaptive hybridization of incentives promotes cooperation. Journal of the Royal Society Interface, 12(102), 2014093520140935.

[23] Wang, Y., Nakao, A., Vasilakos, A. V., \& Ma, J. (2011). P2P soft security: On evolutionary dynamics of P2P incentive mechanism. Computer Communications, 34(3), 241-249.

[24] Buragohain, C., Agrawal, D., \& Suri, S. (2003). A game theoretic framework for incentives in P2P systems. International Conference on Peer-ToPeer Computing (pp.48-56). IEEE Computer Society.

[25] Feldman, M., Lai, K., Stoica, I., \& Chuang, J. (2004). Robust incentive techniques for peer-to-peer networks. ACM Conference on Electronic Commerce (Vol.5, pp.264-267). ACM.

[26] Gupta, R., \& Somani, A. K. (2005). Game Theory As A Tool To Strategize As Well As Predict Nodes Behavior In Peer-to-Peer Networks. International Conference on Parallel and Distributed Systems (Vol.1, pp.244 - 249 Vol. 1). IEEE Computer Society.

[27] Liu, Y., Zhang, J., An, B., \& Sen, S. (2015). A simulation framework for measuring robustness of incentive mechanisms and its implementation in reputation systems. Autonomous Agents and Multi-Agent Systems, 30(4), $1-20$. 
[28] Liu, Y., Zhang, J., An, B., \& Sen, S. (2015). A simulation framework for measuring robustness of incentive mechanisms and its implementation in reputation systems. Autonomous Agents and Multi-Agent Systems, 1-20.

[29] Jin, X., Li, M., Cui, G., Liu, J., Guo, C., Gao, Y., \& Tan, X. (2015) RIMBED: Recommendation Incentive Mechanism Based on Evolutionary Dynamics in P2P Networks. 2015 24th International Conference on Computer Communication and Networks (ICCCN), 1-8.

[30] Jorgen W Weibull. (1997) Evolutionary game theory. MIT press.

[31] Wang, Y., Nakao, A., \& Vasilakos, A. V. (2012). Heterogeneity playing key role: modeling and analyzing the dynamics of incentive mechanisms in autonomous networks. Crop \& Pasture Science, 7(3), 51-53.

[32] Zhao, B. Q., Lui, J. C. S., \& Chiu, D. M. (2012) A mathematical framework for analyzing adaptive incentive protocols in P2P networks. IEEE/ACM Transactions on Networking, 20(2), 367-380.

[33] Park, S., Chung, H., Lee, C., Lee, S., \& Lee, K. (2015). Methodology and implementation for tracking the file sharers using BitTorrent. Multimedia Tools and Applications, 74(1), 271-286.

[34] Wang, Y., Vasilakos, A. V., \& Ma, J. (2015) VPEF: a simple and effective incentive mechanism in community-based autonomous networks. IEEE Transactions on Network \& Service Management, 12(1), 75-86.

[35] Wang, Y., Nakao, A., Vasilakos, A. V., \& Ma, J. (2011). Overview of Modeling and Analysis of Incentive Mechanisms Based on Evolutionary Game Theory in Autonomous Networks. Autonomous Decentralized Systems (ISADS), 2011 10th International Symposium on 659-664.

[36] Alberto A., \& Marco T. (2011) Network fluctuations hinder cooperation in evolutionary games. Plos One, 6(10), e25555.

[37] Xia, C., Wang, J., Wang, L., Sun, S., Sun, J., \& Wang, J. (2012) Role of update dynamics in the collective cooperation on the spatial snowdrift games: beyond unconditional imitation and replicator dynamics. Chaos Solitons \& Fractals, 45(9), 1239-1245. 
[38] Mao, Y., Zhu, P., \& Wei, G. (2013). A game theoretic model for wireless sensor networks with hidden-action attacks. International Journal of Distributed Sensor Networks, 2013(3), 141-149.

[39] Tang, C., Li, A., \& Li, X. (2015). When reputation enforces evolutionary cooperation in unreliable manets. IEEE Transactions on Cybernetics, 45(10), 2190-2201.

[40] Cui, G., Li, M., Wang, Z., Ren, J., Jiao, D., \& Ma, J. (2014). Analysis and evaluation of incentive mechanisms in P2P networks: a spatial evolutionary game theory perspective. Concurrency \& Computation Practice \& Experience, 27(12), 3044-3064.

[41] Josef Hofbauer \& Karl Sigmund (1998) Evolutionary games and population dynamics. Cambridge University Press.

[42] Hisashi Ohtsuki \& Martin A. Nowak (2006) The replicator equation on graphs. Journal of Theoretical Biology, 243(1):86-97.

[43] Mohammed Onimisi Yahaya (2015) Free Riding in Peer-to-Peer Networks: Review and Analysis. Afr J. of Comp \& ICTs, 8(1): 53-60. 